\title{
Clinical Usefulness of Digital Radiography in the Gastrointestinal Tract: Efficacy of Magnification Method
}

\author{
Yoshinori Sugino, Yutaka Imai, Hirohisa Fujisawa, Kyouichi Hiramatsu, Hiroshi Amoh, \\ and Kenji Kumakura
}

\begin{abstract}
We assessed the performance capabilities of image intensifier digital radiography (II DR) in the detection of minute lesions in patients with early stomach cancer. The DR system was a prototype II DR system developed by Toshiba Corp (Tokyo, Japan). This system was able to acquire images with a 1,024- $x$ 1,024-pixel matrix and 12 bits. Radiography was performed using a 0.3-mm tube focus. For the detectability of early stomach cancer, DR was judged to be superior to conventional screen-film system (CFSS) (DR superior, $55.7 \%$; CFSS superior, $22.6 \%$ ). In depicting the characteristics of the surface of the lesion, DR was also judged to be superior to CFSS (DR superior, $56.6 \%$; CFSS superior, $17.0 \%$ ). The II DR system used in this study was able to achieve almost the same spatial resolution as conventional radiography using the magnification method. It was also able to visualize subtle findings of early gastric cancer more clearly by the use of postprocessing. In addition, II DR has the advantages of reducing the patient exposure dose and permitting the acquisition of real-time images. Copyright $₫ 1995$ by W.B. Saunders Company
\end{abstract}

KEY WORDS: image intensifier digital radiography (II DR], gastrointestinal tract, magnification, early gastric cancer.

C OMPUTED RADIOGRAPHY (CR) using imaging plates was the first method used for digitization of $\mathrm{x}$-ray images of the gastrointestinal (GI) tract. However, as a result of improvement of image intensifier (II) technology, the development of high-quality television (TV) systems and progress in electronics, digital radiography (DR) using an II was recently received considerable attention. ${ }^{1,2}$ It is expected that II DR will supplant the conventional filmscreen system (CFSS) when its spatial resolution reaches a level where the GI tract can be examined in fine detail. DR provides a number of functions that are extremely useful in the

From the Department of Radiology, School of Medicine, Keio University; the Department of Radiology, Yamato City Hospital; and the Department of Radiology, School of Medicine, Tokai University, Tokyo, Japan.

Address reprint requests to Yoshinori Sugino, MD, Department of Radiology, School of Medicine, Keio University, 35 Shinanomachi, Shinjuku-ku, Tokyo 160, Japan.

Copyright $\odot 1995$ by W.B. Saunders Company

0897-1889/95/0801-1018\$3.00/0 x-ray examination of the GI tract, such as real-time image acquisition, sequential exposure, and so on.

Therefore, in this study, we assessed the performance capabilities of II DR in the detection of minute lesions in patients with early stomach cancer.

\section{MATERIALS AND METHODS}

\section{Equipment}

The DR system used in this study was a prototype II DR system developed by Toshiba Corp (Tokyo, Japan). This system is able to acquire images with a 1,024- $\times 1,024$-pixel matrix and 12-bit density resolution (4,096 gradations). The system includes a Toshiba DAW $10 \mathrm{~A}$ remote-controlled gastric table, a Toshiba DXB0335CS $\mathrm{x}$-ray tube (tube focus sizes: $0.3 \mathrm{~mm} / 0.8 \mathrm{~mm}$ ), an Advanced Super Metal II image intensifier (field sizes: $12 / 9 / 5$ in), and a Saticon TV camera with 1,051 scanning lines (all from Toshiba Corp, Tokyo, Japan). Radiography was performed using a $0.3-\mathrm{mm}$ tube focus at a tube voltage of 86 kilovolt (peak) and a tube current of $100 \mathrm{~mA}$. A Fuji (Tokyo, Japan) LP-LP 2636 laser printer was used generate hard copies. CFSS radiography was performed at a tube voltage of $100 \mathrm{kVp}$ and a tube current of $320 \mathrm{~mA}$ using the diagnostic table described above, Fuji RX film (standard type), a Kyokko (Tokyo, Japan) BM-III intensifying screen, and a 0.8-mm tube focus.

\section{Modification of the Equipment and Magnification Methods}

The diagnostic table used in this study was an upgraded under-table tube table provided with two grids (Fig 1): a high-ratio (16:1) for radiography and a low-ratio grid (6:1) for fluoroscopy. This upgrade was obtained to permit acquisition of sharp images with less fogging caused by scattered radiation using CFSS and to permit reduction of the exposure dose during fluoroscopy. ${ }^{3,4}$

For DR studies, the $0.3-\mathrm{mm}$ focus was selected because the low-ratio grid for fluoroscopy was used, the capacity of the $\mathrm{x}$-ray tube was sufficiently high, and magnification radiography can be performed without performing special procedures; the spot film device is simply moved farther away from the patient on the under-table tube table.

\section{Measurement of Basic Parameters}

Surface dose measurement during radiography in DR and CFSS studies were obtained using a dosimeter (Model 1015 Radiation Monitor; Radcal Corp \& Co, Monrovia, CA) and an acrylic phantom (6- to 22-cm thick).

DR was performed using the $6: 1$ grid (aluminum, 42 line pairs $/ \mathrm{cm}$ ) with a radiographic tube current of $80 \mathrm{~mA}$ and a 

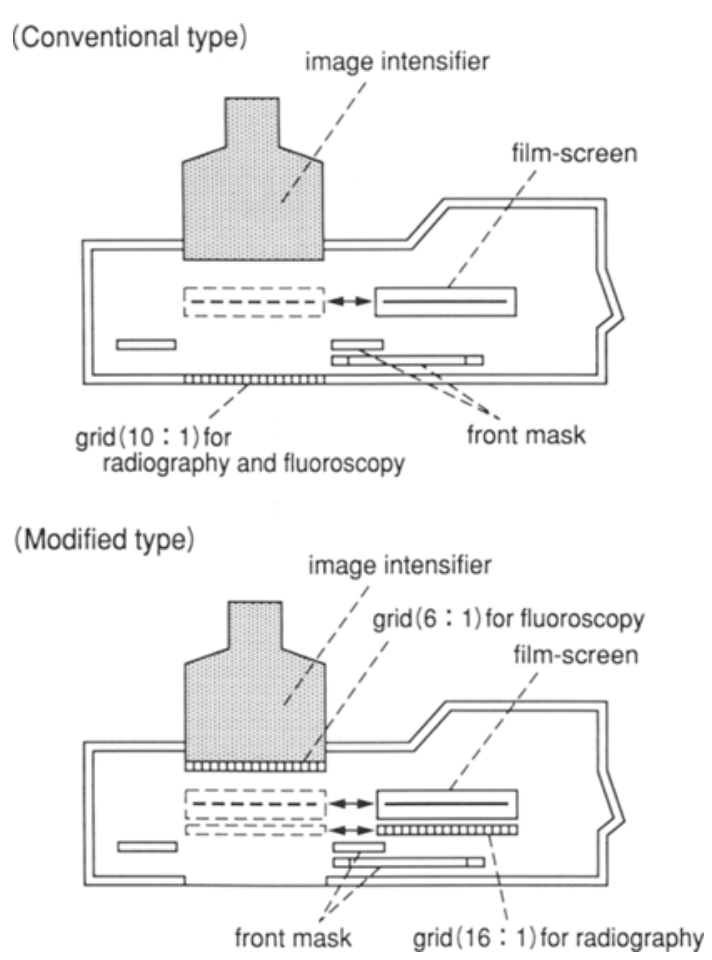

Fig 1. Modification of diagnostic table. The spot device of the under-table tube was modified to have two grid systems. The high-ratio grid (16:1), which is movable, is equipped just in front of the film-screen device for radiography. The low-ratio grid $(6: 1)$ was settled in front of the image intensifier for fluoroscopy.

radiographic tube voltage of $86 \mathrm{kVp}$. CFSS studies were performed by two methods: (1) using the 10:1 grid (aluminum, $42 \mathrm{lp} / \mathrm{cm}$ ) with a radiographic tube current of $300 \mathrm{~mA}$ and a radiographic tube voltage of $86 \mathrm{kVp}$ (standard conditions generally used for radiographic examination of the GI tract), and (2) using the high-ratio 16:1 grid (aluminum, $42 \mathrm{lp} / \mathrm{cm}$ ) with a radiographic tube current of $320 \mathrm{~mA}$ and a high radiographic tube voltage of $100 \mathrm{kVp}$ (selected by authors).

Spatial resolution was measured using a standard chart (x-ray modulation factor transfer function measuring chart: JIS Z4 917-1984 high-voltage chart type M-1W 100R, $100-\mu \mathrm{m}$ tungsten thickness, and 0.5 to $5.0 \mathrm{lp} / \mathrm{mm}$ plus 3.0 to $6.0 \mathrm{lp} / \mathrm{mm})$ and an acrylic phantom. For DR, the tube focus $(0.3 \mathrm{~mm} / 0.8 \mathrm{~mm})$ and field size $(12-/ 9-/ 5$-in $)$ were switched. Magnification radiography, which was said to be effective for improving spatial resolution, was also assessed.

CFSS radiography was performed using the $0.8-\mathrm{mm}$ tube focus at a magnification ratio of 1.17 for clinical application.

\section{Clinical Evaluation}

Of patients with early stomach cancer who underwent resection at Keio University Hospital between September 1990 and March 1993, 103 patients with 106 lesions (21 elevated lesions and 85 depressed lesions) for whom DR

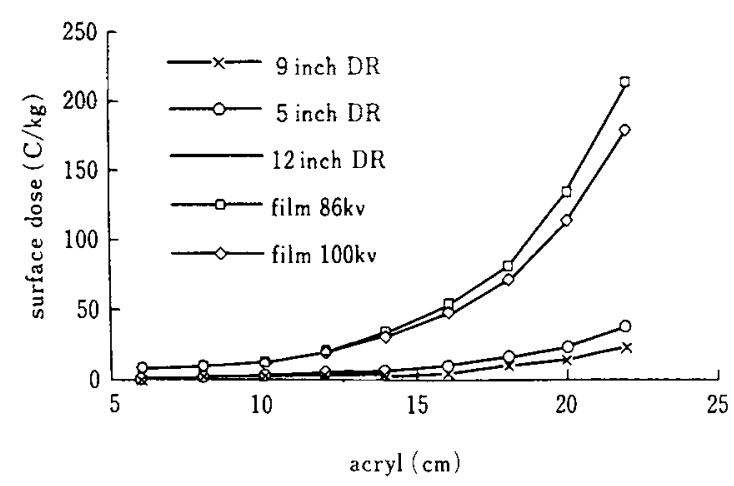

Fig 2. Surface dosage per one radiograph with DR and CFSS. The transverse axis shows the thickness of acryl phantom, and the longitudinal axis shows the surface dosage.

and CFSS images were obtained before surgery were included in the present study.

DR and CFSS were compared with regard to their ability to depict the contour of the lesion, the characteristics of the surface of the lesion, and the condition of the surrounding gastric mucosa. Five GI radiologists served as judges.

\section{RESULTS}

\section{Patient Exposure}

As shown in Fig 2, the surface dose in DR studies was $20 \%$ to $30 \%$ lower than that in CFSS studies, and the surface dose did not exceed one half even when magnification radiography (described below) was performed.

\section{Spatial Resolution}

In DR studies, when the magnification ratio exceeded 1.4 and $0.3-\mathrm{mm}$ tube focus and a 5 -in II field were used, the spatial resolution was greater than $4.0 \mathrm{lp} / \mathrm{mm}$, which is generally

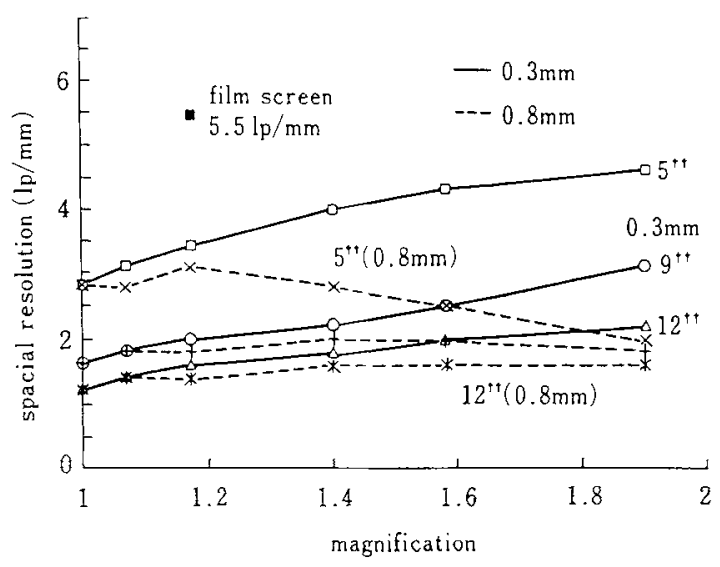

Fig 3. Spatial resolution of DR and CFSS. The transverse axis shows the magnification ratio and the longitudinal axis shows the number of line pairs discernible per millimeter. 


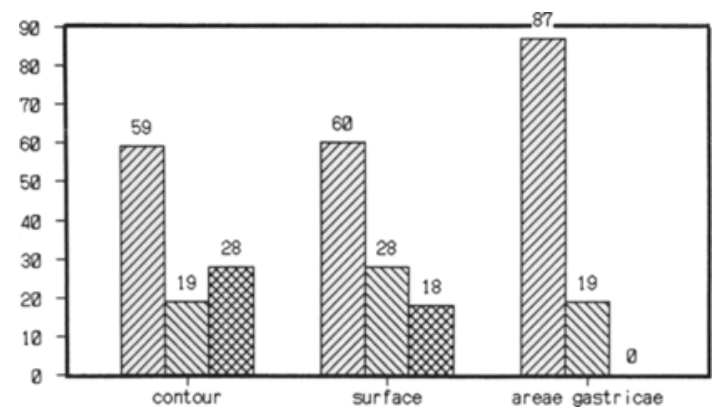

Fig 4. Comparison of clinical images between DR and CFSS. One hundred six lesions of early stomach cancer were studied regarding the visualization of the contour and surface

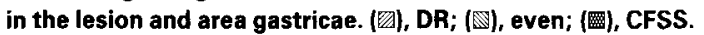

considered a standard value for $\mathrm{x}$-ray diagnostic tables used for GI studies (Fig 3). Spatial resolution was $5.5 \mathrm{lp} / \mathrm{mm}$ for CFSS studies performed using the upgraded diagnostic table used in this study.

\section{Clinical Evaluation}

With regard to the detectability of early stomach cancer (Fig 4), DR was judged to be superior to CFSS (DR superior, 55.7\%; CFSS superior, $22.6 \%$ ). In depicting the characteristics of the surface of the lesion, DR was also

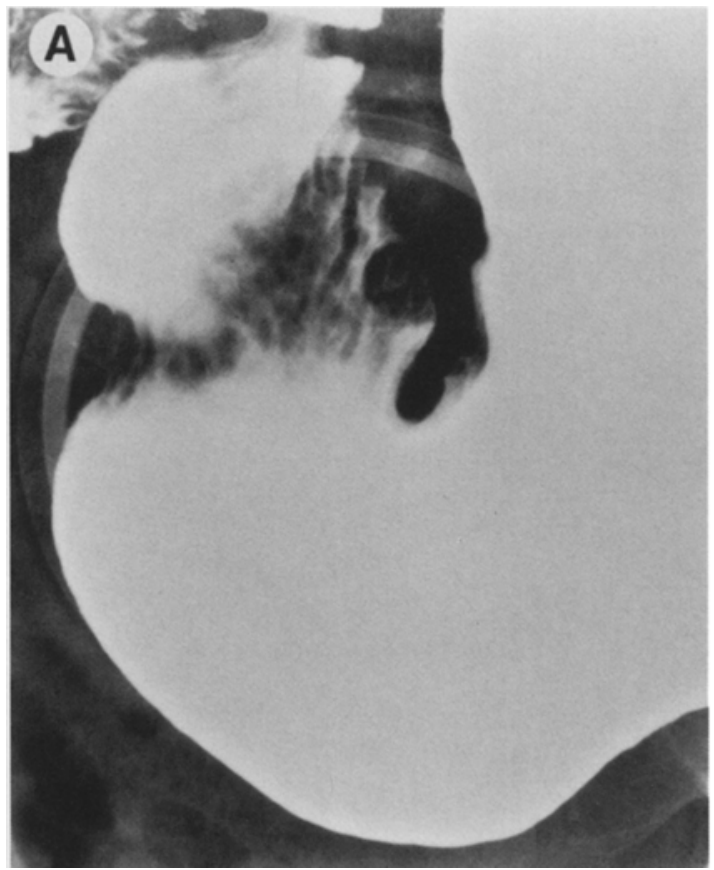

judged to be superior to CFSS (DR superior, $56.6 \%$; CFSS superior, $17.0 \%$ ). In addition, in assessing the gastric mucosa surrounding the lesion, DR was judged to be far superior to CFSS (DR superior, $82 \%$; equal, $18 \%$; CFSS superior, $0 \%$ ).

\section{DISCUSSION}

Reduction of patient dose, real-time image acquisition, sequential exposure, etc are the advantages of II DR. However, in the past, the spatial resolution of DR was inferior to that of CFSS and of CR using imaging plate. In this study, DR was found to be superior in the detection of early stomach cancer though its spatial resolution remained inferior to that of CFSS.

The reason for this result are thought to be as follows. In DR studies, radiographic failures caused by blurring due to patient motion or inappropriate radiographic conditions can be eliminated because radiographic images can be observed in real time. In addition, DR permits fine details to be clearly displayed by postprocessing (Fig 5).

Magnification radiography using DR is effec-

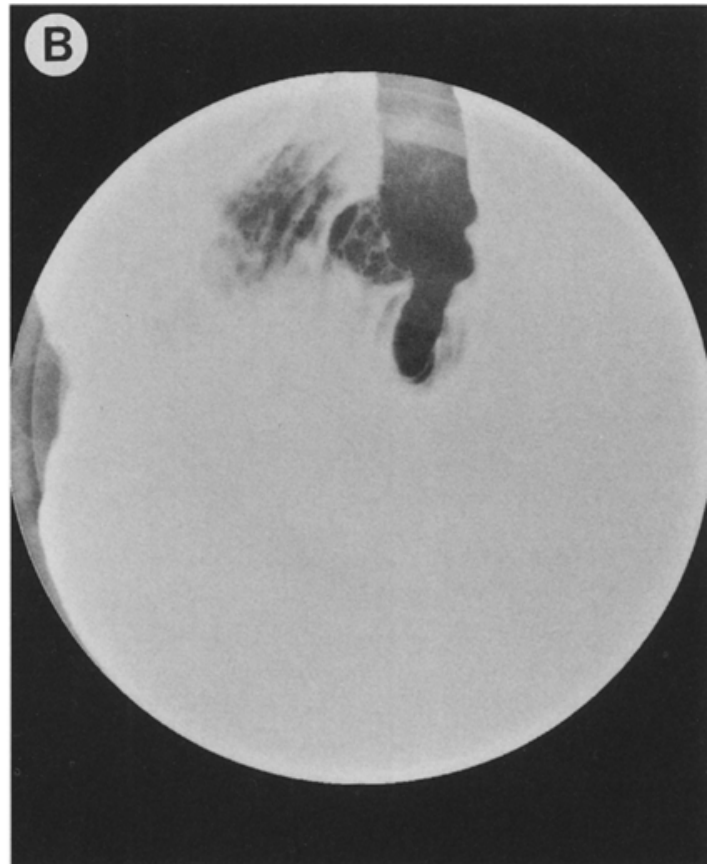

Fig 5. Images of compression study in a patient with early stomach cancer in the atrium (superficial elevation with central depression [lla + Ilc type], $1.0 \mathrm{~cm}$ in diameter, histologically well-differentiated tubular adenocarcinoma confined to the mucosal layer). In comparison with CFSS (A), fine granurality on the lesion was well visualized in the postprocessed DR images (B). 


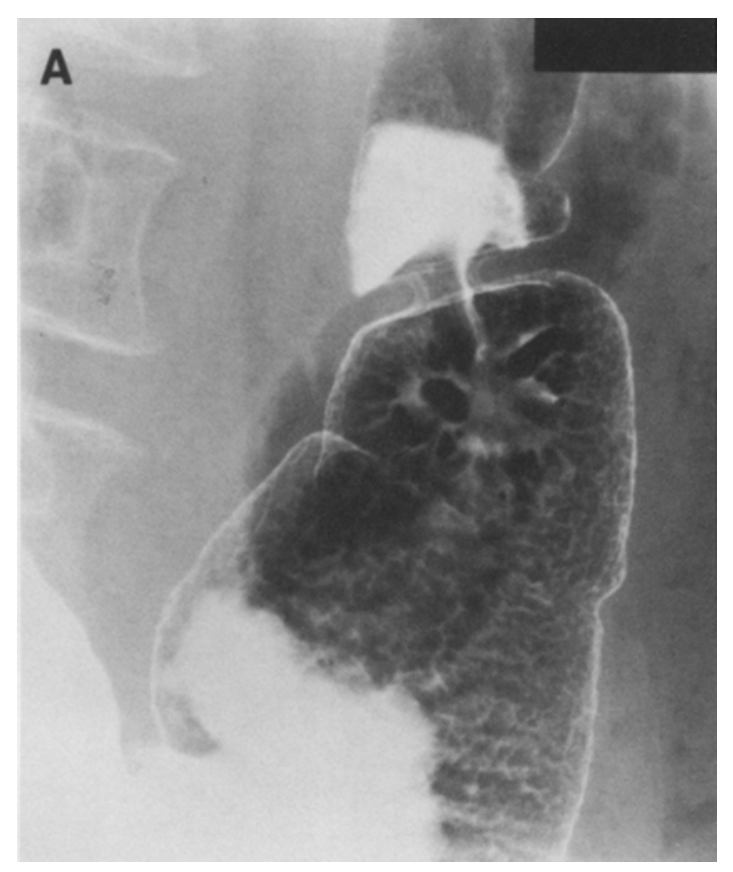

Fig 6. Images of double-contrast study in a patient with early stomach cancer in the prepyloric portion (superficial depression [IIc type], $2.7 \mathrm{~cm}$ in diameter, histologically moderately differentiated adenocarcinoma confined to the mucosal layer). (A), CFSS image; (B) DR image with 5-in II field; (C), DR image with 5-in II field with magnification method. The DR image with magnification depicted the fine details of the irregular contour more clearly than other two images.

tive in improving spatial resolution. ${ }^{5}$ Nakano et $\mathrm{al}^{6}$ have studied magnification radiography for the gastrointestinal tract using CR. Their method involves rather complicated procedures because radiography is performed using two $\mathrm{X}$-ray tubes, and the patient must be repositioned. In our method, magnification radiography can be performed easily using the under-table tube table by simply moving the spot film device farther away from the patient. Procedures are easier than CFSS studies because the need to set the spot film device close to the patient is eliminated. At present, a magnification ratio of $\sim 1.6$ is available by setting the spot film device to the maximal distance from the patient.

The spatial resolution of $5.5 \mathrm{lp} / \mathrm{mm}$ for CFSS obtained in this study is superior to that achieved by conventional systems used by GI studies.
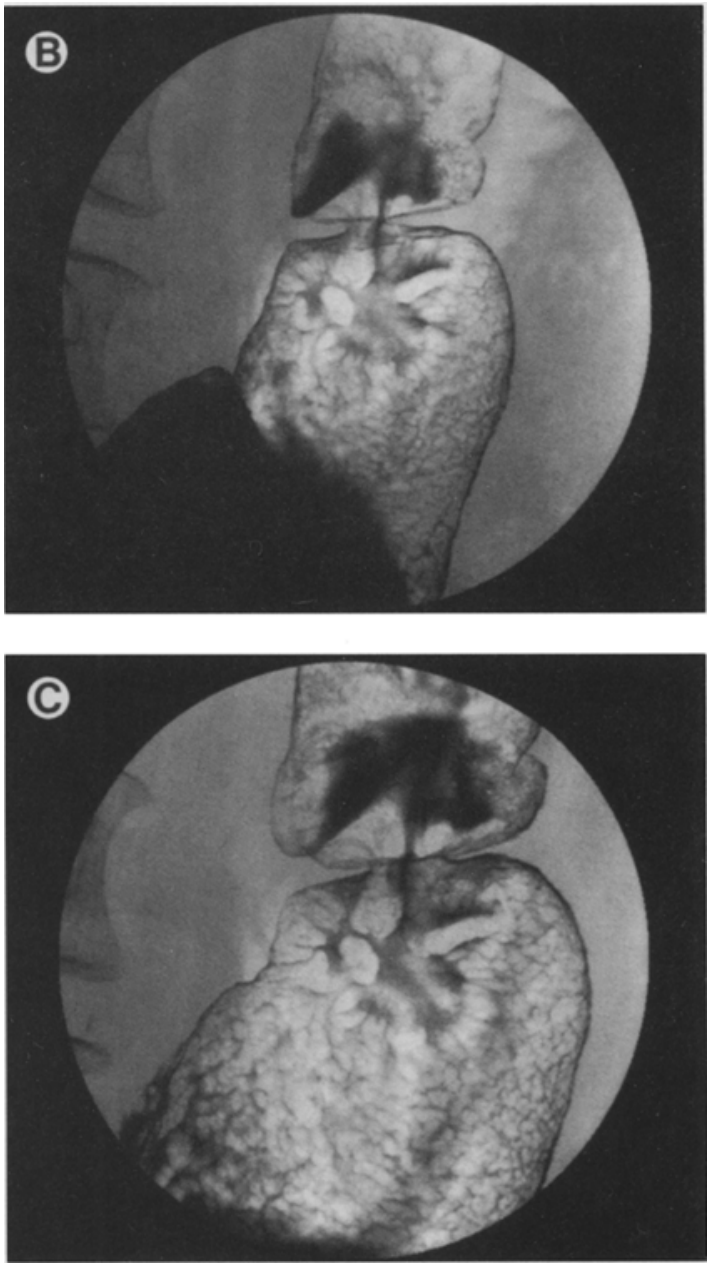

With regard to the detection of gastric lesions, mucosal areas smaller than an area gastrica can be detected. ${ }^{1,7}$ However, the spatial resolution of CFSS using a general gastric table is $\sim 4.0$ $\mathrm{lp} / \mathrm{mm}$, which was almost equivalent to that achieved by magnification radiography using DR in the present study (Fig 6).

In the future, one method for improving the spatial resolution of DR images will be increasing the matrix size, and a model with a matrix size of $2,048 \times 2,048$ pixels has already been introduced. However, there are still a number of problems remaining; devices such as the cathode-ray tube are expensive, storage and management of data (four times larger for a $2,048-\times 2,048$-pixel image than a 1,024- $\times$ 1,024-pixel image) is troublesome, and the exposure dose must be increased to reduce noise. 


\section{CONCLUSION}

The II DR system used in this study was able to achieve almost the same spatial resolution as conventional radiography using the magnification method. It was also able to visualize subtle findings of early gastric cancer more clearly by the use of postprocessing. In addition, II DR has the advantages of reducing the patient exposure dose and permitting the acquisition of real-time images.

\section{REFERENCES}

1. Okuda S, Sugino Y, Imai Y, et al: Image intensifier digital radiography of colorectal lesion. Jpn J Clin Radiol 38:231-239, 1993

2. Takahashi M, Ueno S, Yoshimatsu S, et al: Gastrointestinal examination with digital radiography. Radiographics 12:969-978, 1993

3. Sugino Y: Evaluation of anti-scatter grids for barium study of the stomach. Nippon Acta Radiol 48:1349-1363, 1988

4. Kumakura K, Sugino Y, Baba Y: X-ray equipment, in: Radiology of the Stomach. Tokyo, Japan, Kanehara \& Co, 1992 pp 1-23
5. Curry TS III, Dowdey JE, Murry RC Jr: Digital radiography, in: Christensen's Physics of Diagnostic Radiology (ed 4). Philadelphia, PA, Lea \& Febiger, 1990 pp $392-431$

6. Nakano $Y$, Togashi $K$, Torizuka K, et al: Stomach and duodenum: Radiographic magnification using computed radiography (CR). Radiology 160:383-387, 1986

7. Sugino Y, Kumakura K: Radiological diagnosis of flat type (type II b) early gastric cancer: A new means of evaluation. Gastroenterological Endoscopy 32:2471-2474, 1990 